\title{
CURCULIÓNIDOS DE DIVERSAS LOCALIDADES DE LA REPÚBLICA MEXICANA (COLEOPTERA: CURCULIONOIDEA)
}

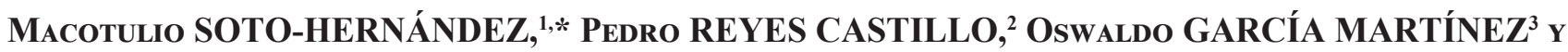 \\ SALVADOR ORDAZ SILVA ${ }^{4}$
}

\author{
${ }^{1}$ Instituto Nacional de Investigaciones Forestales, Agrícolas y Pecuarias. Sitio Experimental Zaragoza, Coahuila. \\ *Autor para correspondencia: <gmrkoz_2000@hotmail.com> \\ ${ }^{2}$ Instituto de Ecología, A.C. Carretera Antigua a Coatepec 351, El Haya, 91070 Xalapa, Veracruz. México. \\ <pasalidos@hotmail.com> \\ ${ }^{3}$ Universidad Autónoma Agraria Antonio Narro, Departamento de Parasitología Agrícola. Buenavista, Saltillo, \\ Coahuila. México. C.P. 25315.<drogarcia@yahoo.com.mx> \\ ${ }^{4}$ Facultad de Ingeniería y Negocios, Universidad Autónoma de Baja California, Km 180.2, Carretera Ensenada- \\ San Quintín, Ejido Padre Kino, C.P. 22930, San Quintín, Baja California, México.<ordaz_silva@hotmail.com> \\ Recibido: 22/05/2015; aceptado: 26/01/2016
}

\begin{abstract}
Soto-Hernández, M., Reyes-Castillo, P., García-Martínez, O. \& Ordaz-Silva, S. 2016. Curculiónidos de diversas localidades de la República Mexicana (Coleoptera: Curculionoidea). Acta Zoológica Mexicana (n. s.), 32(1): 62-70.
\end{abstract}

RESUMEN. Se realizaron colectas en diversas localidades de siete Estados de la República Mexicana en los años 2010-2014. Se identificaron un total de 77 especies en 50 géneros. De los cuales, Onychylis texanus Burke es nuevo registro para México; además para Coahuila, Anthonomus tenuis; Veracruz, Anthonomus fischeri, Anthonomus schwarzi, Atractomerus recessus; Jalisco, Anthonomus maltanza, Pantomorus picturatus; Guerrero, Embates ocellatus, Optatus palmaris, Rodobaenus mundus y para Michoacán, Sibinia dissipata. Por su hábito alimenticio, algunas especies son consideradas de importancia económica: Cactophagus validirostris, Metamasius hemipterus, Rhodobaenus sanguineus, Scyphophorus acupunctatus, Rhinostomus barbirostris, Rhynchophorus palmarum, Optatus palmaris, Cylindrocopturus biradiatus, Epicaerus aurifer, E. mexicanus, Exophthalmus opulentus, Sphenophorus coesifrons,Metamasius callizona, M. hemipterus, M. sellatus y Pantomorus godmani.

Palabras clave: Picudos, nuevos registros, distribución, importancia económica.

\section{INTRODUCCIÓN}

México es uno de los países con mayor diversidad biológica, aunque la verdadera riqueza específica que existe en el país aún es desconocida, particularmente en artrópodos; Phylum que contiene el mayor número de especies conocidas con un $85 \%$ del total de la fauna mundial (Llorente-Bousquets et al. 1996). Dentro del reino animal, el orden Coleoptera es el más grande y diverso en el planeta, y donde Curculionoidea representa uno de los grupos con mayor número de especies. Para México, actualmente se
Soto-Hernández, M., Reyes-Castillo, P., García-Martínez, O. \& Ordaz-Silva, S. 2016. Weevils collected in diverse localities of Mexico (Coleoptera: Curculionoidea). Acta Zoológica Mexicana (n. s.), 32(1): 62-70.

ABSTRACT. Collecting was done on diverse localities of seven States of Mexico from years 2010 to 2014 . A total of 77 species of 50 genera were identified, from which, Onychylis texanus Burke is a new record for Mexico; furthermore several are new record for some Mexican states: Coahuila Anthonomus tenuis; Veracruz, Anthonomus fischeri, Anthonomus schwarzi, Atractomerus recessus; Jalisco, Anthonomus maltanza, Pantomorus picturatus; Guerrero, Embates ocellatus, Optatus palmaris, Rodobaenus mundus and for Michoacán, Sibinia dissipata. Some weevils are considered of economic importance for some crops: Cactophagus validirostris, Metamasius hemipterus, Rhodobaenus sanguineus, Scyphophorus acupunctatus, Rhinostomus barbirostris, Rhynchophorus palmarum, Optatus palmaris, Cylindrocopturus biradiatus, Epicaerus aurifer, E. mexicanus, Exophthalmus opulentus, Sphenophorus coesifrons, Metamasius callizona, M. hemipterus, $M$. sellatus and Pantomorus godmani.

Key words: Weevils, distribution, new records, economical importance.

reconocen 3594 especies de curculiónidos (sensu lato), de las cuales aproximadamente $40 \%$ son consideradas endémicas (Morrone 2014).

Anderson \& O'Brien (1996), citan que, se conoce menos del $50 \%$ de los curculiónidos mexicanos, entre las limitantes para su estudio se encuentran la falta de claves taxonómicas para separar la mayoría de los grupos, las principales colecciones de referencia están en el extranjero y, además las colectas han sido esporádicas y localizadas. Actualmente para el país, sin duda la principal referencia para identificar la mayoría de los curculiónidos 
aún sigue siendo la Biologia Centrali-Americana (Sharp \& Champion 1889-1911, Champion 1902-1906, 19091911) y algunas publicaciones de Anderson (2002), Kissinger (1964) y Vaurie (1951, 1966, 1967, 1978, 1981). Ha habido avances en el estudio de algunos grupos, principalmente los de importancia económica.

Por lo anterior, el objetivo del presente trabajo es presentar una lista de especies de curculiónidos recolectadas en diversas localidades de México, además de una sinopsis de su distribución geográfica.

\section{MATERIALES Y MÉTODOS}

Se realizaron recolectas en diversas localidades de Coahuila, Guerrero, Jalisco, Puebla, Querétaro, San Luis Potosí y Veracruz durante los años 2010-2014. La mayoría de los ejemplares se capturaron con ayuda de una sombrilla entomológica de $1 \mathrm{~m}^{2}$, la cual, se colocó debajo del follaje de las plantas, mismas que fueron sacudidas con una vara de $1 \mathrm{~m}$ de longitud aproximadamente con el fin de provocar la caída de los curculiónidos en la sombrilla, los especímenes se recogían, y se sacrificaron en un frasco con acetato de etilo, después se mantuvieron en microtubos Eppendorf para su traslado al laboratorio.

Todos los especímenes recolectados se montaron en alfileres entomológicos. La identificación se hizo utilizando claves dicotómicas de los siguientes autores: Sharp \& Champion (1889-1911), Champion (1902-1906, 19091911), Anderson (2002, 2002a), Clark (1978, 1987, 1989, 1990, 1993), Clark \& Burke (2005), Soto-Hernández et al. (2013), O’Brien \& Wibmer (1990), Prena (2005, 2009), Kissinger (1964, 1970), Rosas (2011) y Vaurie (1951, 1966, 1967, 1978, 1981). Los especímenes identificados se compararon con ejemplares depositados en la Colección Nacional de Insectos (CNIN), Colección Entomológica de la Facultad de Ciencias Naturales, Universidad Autónoma de Querétaro (UAQE), en las base de datos: Franz et al. (2012) Southwest Collections of Artropods Network (SCAN), Fred (2009) Entomological Guyane, Perkins (2010) M.C.Z Type Database Harvard Entomology y Barrigan-Tuñon (2009) Coleoptera Neotropical. Se revisaron los listados de O'Brien \& Wibmer (1882, 1984). La clasificación está basada en Alonso-Zarazaga \& Lyal (1999). Los especímenes fueron depositados en la Colección de Insectos IEXA, Xalapa, Veracruz, Colección Nacional de Insectos (CNIN) México D.F., y Facultad de Ciencias Naturales de la Universidad Autónoma de Querétaro (UAQE).

\section{RESULTADOS}

Fueron identificadas cinco familias de curculiónidos: Attelabidae, Brentidae, Dryophthoridae, Erirhinidae y Curculionidae, un total de 77 especies fueron determinadas en 50 géneros. De las cuales, Dryophthoridae y Curculionidae registraron el mayor número de especies identificadas, 17 y 56 respectivamente. En Dryophthoridae se identificaron dos subfamilias y tres tribus, mientras que en Curculionidae ocho subfamilias y 26 tribus, siendo Anthonomini y Naupactini con el mayor número de especies con ocho y seis respectivamente.

De las especies identificadas, Onychylis texanus Burke (Burke, 1959) es nuevo registro para México. Además se registran para Coahuila, Anthonomus tenuis Fall 1913; Veracruz, Anthonomus fischeri Blackwelder 1947, Anthonomus schwarzi Clark \& Burke 1985, Atractomerus recessus Clark 1989; Jalisco, Anthonomus maltanza Clark 1988, Pantomorus picturatus Sharp 1891; Guerrero, Embates ocellatus (Champion 1907), Optatus palmaris (Pascoe 1889), Rhodobaenus mundus (Champion 1910) y para Michoacán, Sibinia dissipata Champion 1910. Estos nuevos registros se indican en la lista por un asterisco $(*)$.

\section{Lista de especies de Curculionoideae \\ Familia Attelabidae \\ Subfamilia Attelabinae \\ Tribu Attelabini}

Xestolabus corvinus (Gyllenhal, 1839). México: Veracruz: Jalcomulco, 09-I-2014. Colectado en Tillandsia sp., colector Adriana Hernández.

Distribución: Belice, Costa Rica, Guatemala, Honduras, México, Nicaragua, Panamá, Sudamérica.

Hybolabus ater (Olivier, 1789). México: Puebla: Cuetzalan, 24-VII-2012. Colector Omar Villerías.

Distribución: Guatemala, México, Sudamérica.

Familia Brentidae

Subfamilia Brentinae

Tribu Brentini

Brentus anchorago Linnaeus, 1758. México: Veracruz: Jalcomulco, 30-I-2014. Colectado en Tillandsia sp., colector Adriana Hernández. México: Guerrero: Cuetzala del Progreso, Chilacachapa, 25-IV-2014. Colectado en tronco de Ficus sp., colector Omar Villerías.

Distribución: Estados Unidos de América, Guadalupe, Dominica, México, Belice, Guatemala, El Salvador, Nicaragua, Costa Rica, Panamá, Brasil, Paraguay, Argentina.

Familia Dryophthoridae 
Subfamilia Rhynchophorynae

Tribu Sphenophorini

Cactophagus validirostris (Gyllenhal, 1838). México: Veracruz: Xalapa, Las Ánimas, 09-V-2005. 1260 msnm. Colector Ma. Luisa Castillo. México: Veracruz: Chocaman, 11-XII-2012, $18^{\circ}$ 59' 03' 'N, 097 01' 02'’O. Colector Macotulio Soto $\mathrm{H}$.

Distribución: Guatemala, Honduras, México, Panamá, Sudamérica.

Metamasius callizona (Chevrolat, 1883). México: Veracruz: Jalcomulco, 02-II-2014. Colectado en Tillandsia limbata, colector Adriana Hernández. México: Veracruz: Jalcomulco, 08-X-2013. Colectado en Tillandsia variabilis, colector Macotulio Soto $\mathrm{H}$.

Distribución: Guatemala, México, Panamá.

Metamasius ensirostris (Germar, 1824). México: Veracruz: Chocaman, 11-XII-2012, $18^{\circ} 59^{\prime} 03^{\prime}$ 'N, 097 01' 02 'O. Colectado en plantación de café, colector Macotulio Soto $\mathrm{H}$.

Distribución: México, Sudamérica

Metamasius hemipterus (Linnaeus, 1758). México: Veracruz: Chocaman, 11-XII-2012. $18^{\circ} 59^{\prime} 03^{\prime}{ }^{\prime} \mathrm{N}, 097^{\circ} 01^{\prime}$ 02'’O. Colectado en plantación de café, colector Macotulio Soto H. México: Veracruz: Jalcomulco, 09-IV-2014. Colectado en Tillandsia spp. (Bromeliaceae), colector Adriana Hernández.

Distribución: Antigua, Barbados, Bequia, Dominica, Granada, Guadalupe, Jamaica, Martinica, Montserrat, Puerto Rico, St. Croix, St. Kitts, Santo Tomas, San Vicente, Belice, El Salvador, Guatemala, Honduras, México, Nicaragua, Panamá, Sudamérica.

Metamasius sellatus Champion, 1910. México: Veracruz: Jalcomulco, 09-IV-2014. Colectado en Tillandsia variabilis (Bromeliaceae), colector Macotulio Soto $\mathrm{H}$.

Distribución: Belice, Estados Unidos de América, Costa Rica, Guatemala, México, Nicaragua, Panamá.

Rhodobaenus adspersus (Gyllenhal, 1838). México:

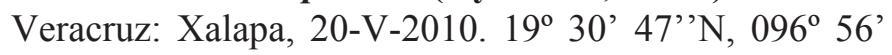
27' 'O. Colector Macotulio Soto H.

Distribución: Guatemala, Honduras, México, Nicaragua, Panamá.

Rhodobaenus auctus Chevrolat, 1885. México: Guerrero: Cuetzala del Progreso, Chilacachapa, 01-IX-2012. Selva baja caducifolia, colector Omar Villerías.

Distribución: Belice, Costa Rica, El Salvador, Guatemala, Honduras, México, Nicaragua, Panamá, Sudamérica.

Rhodobaenus deltoides Chevrolat, 1885. México: Puebla: Cuetzalan, 24-VII-2012. Colector Omar Villerías.

Distribución: Belice, Costa Rica, El Salvador, Guatema- la, Honduras, México, Nicaragua.

Rhodobaenus biundulatus Champion, 1910. México: Veracruz: Xalapa, 20-V-2010, 19 $30^{\prime}{ }^{\prime} 47^{\prime}$ 'N, 096 ${ }^{\circ} 56^{\prime}$ 27 ' O. Colector Macotulio Soto H.

Distribución: México.

Rhodobaenus mundus (Champion, 1910). México: Guerrero*: Cuetzala del Progreso, Chilacachapa, 14-VII2012. Colector Omar Villerías.

Distribución: México (Durango, Guanajuato, Oaxaca, Veracruz).

Rhodobaenus pustulosus (Gyllenhall, 1838). México: Guerrero: Cuetzala del progreso, Chilacachapa, 01-IX2012. Selva baja caducifolia, colector Omar Villerías.

Distribución: Estados Unidos de América, Costa Rica, Guatemala, México, Nicaragua, Panamá.

Rhodobaenus sanguineus (Gyllenhal, 1838). México: Guerrero: Cuetzala del Progreso, 24-VII-2012. Col. Omar Villerías. Veracruz, Acajete, 12-VII-2010, 19³ $34^{\prime} 51^{\prime \prime} \mathrm{N}$, $097^{\circ}$ 00' 42' 'O. 1,960 msnm. Colector Macotulio Soto $\mathrm{H}$.

Distribución: Costa Rica, El Salvador, Guatemala, México, Nicaragua, Panamá.

Rhodobaenus stigmaticus (Fahraeus, 1845). México: Puebla: Cuetzalan, 24-VII-2012. Colector Omar Villerías; Veracruz, Xalapa, 06-VI-2003. 1300 msnm. Colector Ma. Luisa Castillo en suelo.

Distribución: Belice, Costa Rica, Guatemala, México, Nicaragua, Panamá.

Scyphophorus acupunctatus Gyllenhal, 1838. México: Veracruz: Xalapa. Colectado en el Bosque Mesófilo de Montaña, 16-XI-2004. 1310 msnm. Colector Ma. Luisa Castillo.

Distribución: Viejo mundo, Estados Unidos de América, México, Las Antillas, Centro y Sudamérica.

Sphenophorus coesifrons Gyllenhal, 1838. México: Coahuila: Cuatrociénegas, Poza Azul, 06-X-2007. Colector García M. O.

Distribución: Estados Unidos de América, México.

Tribu Rhinostomini

Rhinostomus barbirostris (Fabricius, 1775). México: Veracruz: Emiliano Zapata, Chavarrillo, 01-II-2000. Colector Reyes-Castillo P.

Distribución: Belice, Costa Rica, Guatemala, Honduras, México, Nicaragua, Panamá, Sudamérica.

Subfamilia Rhynchophorinae

Tribu Rhynchophorini

Rhynchophorus palmarum (Linnaeus, 1758). México: Veracruz: VII-1985. Hospedero palma. Colector ReyesCastillo P. 
Distribución: Estados Unidos de América, México, Las Antillas, Centro y Sudamérica.

Familia Erirhinidae

Subfamilia Erirhininae

Tribu Erirhinini

Onychylis texanus Burke, 1959. México: Veracruz: Hidalgotitlán, 27-X-2012. $17^{\circ} 47^{\prime} 36^{\prime}$ 'N, $094^{\circ} 35^{\prime}$ '40'’O.

Colector Macotulio Soto $\mathrm{H}$.

Distribución: Estados Unidos de América (Texas) y México (Veracruz).

Familia Curculionidae

Subfamilia Curculioninae

Tribu Anthonomini

Anthonomus fischeri Blackwelder, 1947. México: Vera-

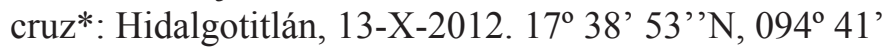
35' 'O. Colector Macotulio Soto H.

Distribución: Brasil, Colombia, Costa Rica, Guatemala, Nicaragua, Panamá, Perú, Venezuela y México (Chiapas, Guerrero, Morelos, Nuevo León, Oaxaca, Tamaulipas).

Anthonomus maltanza Clark, 1988. México: Jalisco*: La Huerta, Estación de Biología Chamela, 01-VIII-2012. $19^{\circ} 29^{\prime} 36^{\prime}$ 'N, $105^{\circ}$ 03' 21' 'O. Colector Macotulio Soto $\mathrm{H}$.

Distribución: México (Sinaloa).

Anthonomus quechpini Clark, 1988. México: Michoacán: Morelia, Cerro del Águila, 20-VII-2013. 19³ $38^{\prime}$ $44^{\prime}$ 'N y $101^{\circ} 20^{\prime} 51^{\prime}$ 'O. 2, 288 msnm. Colector Macotulio Soto $\mathrm{H}$.

Distribución: México (Tamaulipas, Nuevo León, Michoacán).

Anthonomus rufipennis LeConte, 1876. México: Veracruz: Jalcomulco. Arroyo Blanco, $2 \mathrm{~km}$ al SE de Jalcomulco, 17-V-2012. $19^{\circ} 19^{\prime} 27^{\prime} ' \mathrm{~N}, 096^{\circ} 4^{\prime}{ }^{\prime} 52^{\prime}{ }^{\prime} \mathrm{O}$. Colector Macotulio Soto H.

Distribución: Costa Rica, Estados Unidos de América, Guatemala, Honduras, México (Guerrero, Hidalgo, Nuevo León, Oaxaca, Querétaro, Quintana Roo, Sinaloa, Veracruz, Yucatán).

Anthonomus schwarzi Clark \& Burke, 1985. México: Veracruz*: Jalcomulco. Arroyo Blanco, $2 \mathrm{~km}$ al SE de Jalcomulco, 17-V-2012. 19' 19' 27' 'N, 096 ${ }^{\circ} 44^{\prime}$ 52'’O. Colector Macotulio Soto H.

Distribución: Cuba, Estados Unidos de América, Jamaica y México (Campeche, Quintana Roo, Sinaloa, Tamaulipas, Yucatán).

Anthonomus tenuis Fall, 1913. México: Coahuila*: Cuatrociénegas, 13-IX-2007. Colector Roberto García M. O. Distribución: Canadá, Estados Unidos de América y México (Sonora).
Atractomerus recessus Clark, 1989. México: Veracruz*: Hidalgotitlán, 27-X-2012. $17^{\circ} 38^{\prime} 53^{\prime}$ 'N $094^{\circ} 41^{\prime} 35^{\prime}$ 'O. Colector Macotulio Soto $\mathrm{H}$.

Distribución: México (Chiapas).

Lonchophorellus callosus (Faust, 1894). México: Veracruz: Hidalgotitlán, 27-X-2012. $17^{\circ} 38^{\prime} 53^{\prime}$ 'N, 094 41' 35' 'O. Colector Macotulio Soto H.

Distribución: Bolivia, Brasil, Colombia, Costa Rica, E1 Salvador, Guatemala, Honduras, México (Chiapas, Guerrero, Morelos, Puebla, Veracruz), Panamá, Perú, Venezuela.

Tribu Ceratopodini

Ceratopus bisignatus Boheman, 1843. México: Veracruz: Xalapa, 10-V-2010. Colector Bonet A.

Distribución: Belice, Costa Rica, México, Panamá.

Prionobrachium schonherri Faust, 1894. México: Veracruz: Hidalgotitlán, $5.5 \mathrm{~km}$ al NE de Hidalgotitlán, 27-X2012. $17^{\circ} 47^{\prime} 36^{\prime}$ 'N, $094^{\circ} 35^{\prime}$ 40' O. Colector Macotulio Soto $\mathrm{H}$.

Distribución: Salvador, Guatemala, Honduras, México, Panamá, Sudamérica.

Tribu Otidocephalini

Myrmex cylindricollis (Champion, 1903). México: Veracruz: Xalapa, Instituto de Ecología, A. C., 20-VI-2013. Colector Macotulio Soto H.

Distribución: México.

Tribu Piazorhinini

Piazorhinus uniformis (Champion, 1903). México: Jalisco*: La Huerta, Estación de Biología Chamela, Camino Chachalacas, 01-VIII-2012, $19^{\circ} 29^{\prime} 36^{\prime}{ }^{\prime} \mathrm{N}, 105^{\circ} 02^{\prime}$ 21' 'O. Colector Macotulio Soto H.

Distribución: México, Panamá.

Tribu Tychiini

Sibinia dissipata Champion, 1910. México: Michoacán*: Morelia, Cerro del Águila, 20-VII-2013. 19³ 38'44' 'N, $101^{\circ} 20^{\prime} 51^{\prime \prime}$ O. 2, $288 \mathrm{msnm}$. Colector Macotulio Soto $\mathrm{H}$.

Distribución: Nicaragua, México (Guerrero, Puebla, Oaxaca y Sinaloa).

Subfamilia Baridinae

Tribu Ambatini

Embates ocellatus (Champion, 1907). México: Guerrero*: Cuetzala del Progreso, 24-VII-2012. Colector Omar Villerías.

Distribución: Honduras, México (Chiapas, Oaxaca, Tabasco, Veracruz).

Tribu Madopterini

Geraeus hospes (Casey, 1892). México: Michoacán: Morelia, Cerro del Águila, 20-VII-2013, 19³8'44'’N, 
$101^{\circ} 20^{\prime} 51^{\prime \prime}$ O. 2, 288 msnm. Colector Macotulio Soto H. Distribución: Ampliamente distribuida en el sureste de los Estados Unidos de América, México y Centroamérica.

Geraeus modestus (Boheman, 1836). México: Jalisco: La Huerta, Estación de Biología Chamela, 18-VII2013. $19^{\circ} 29^{\prime} 36^{\prime}$ 'N $105^{\circ} 03^{\prime} 21^{\prime}$ 'O. Colector Macotulio Soto $\mathrm{H}$.

Distribución: Estados Unidos de América.

Geraeus pexus (Boheman, 1844). México: Veracruz: Jalcomulco, Arroyo Blanco, $2 \mathrm{~km}$ al SE de Jalcomulco, 17-V-2012. $19^{\circ} 19^{\prime} 27^{\prime}$ 'N, $096^{\circ} 44^{\prime}$ 52' 'O. Colector Macotulio Soto $\mathrm{H}$.

Distribución: Guatemala, México, Nicaragua.

Geraeus tenuispinis Champion, 1908. México: Veracruz: Hidalgotitlán, $5.5 \mathrm{~km}$ al NE de Hidalgotitlán, 27-X2012. $17^{\circ} 47^{\prime} 36^{\prime}$ 'N $094^{\circ} 35^{\prime} 40^{\prime}$ 'O. Colector Macotulio Soto $\mathrm{H}$.

Distribución: Costa Rica, Guatemala, México, Panamá. Geraeus senilis (Ghyllenhal, 1836). México: Guerrero: Cuetzala del Progreso, Chilacachapa, 14-VI-2012. Colector Omar Villerías.

Distribución: Costa Rica, Guatemala, Honduras, México, Nicaragua.

Xystus mexicanus (Boheman, 1844). México: Jalisco: La Huerta, Estación de Biología Chamela, 01-VIII-2012. $19^{\circ}$ 29' 36' 'N. $105^{\circ} 02^{\prime}$ 21' 'O. Colector Macotulio Soto H.

Distribución: Belice, Guatemala, México.

Zygobarella tristicula (Champion, 1908). México: Jalisco: La Huerta, Estación de Biología Chamela, 18-VII2013. $19^{\circ} 29^{\prime} 47^{\prime}$ 'N $105^{\circ} 02^{\prime}$ '24' 'O. Colector Macotulio Soto $\mathrm{H}$.

Distribución: México, Nicaragua y Estados Unidos de América.

Tribu Baridini

Pseudobaris notata (Boheman, 1836). México: Michoacán: Morelia, Cerro del Águila, 20-VII-2013. $19^{\circ} 38^{\prime}$ $44^{\prime}$ 'N, $101^{\circ} 20^{\prime} 51^{\prime}$ 'O. 2,288 msnm. Colector Macotulio Soto $\mathrm{H}$.

Distribución: México.

Eurhinus cupripes (Pascoe, 1886). México: Guerrero: Cuetzala del Progreso, Chilacachapa, 15-V-2012. Colector Omar Villerías.

Distribución: México (Guerrero, Morelos, Puebla, Oaxaca).

Tribu Optatini

Optatus palmaris (Pascoe, 1889). México: Guerrero*: Cuetzala del Progreso, Chilacachapa, 29-VIII-2012. Hospedero Anona, colector Omar Villerías.
Distribución: Honduras, México.

Subfamilia Conoderinae

Tribu Piazurini

Cratosomus punctulatus f. pustulatus Gyllenhal, 1837. México: Guerrero: Cuetzala del Progreso, Chilacachapa, 05-XII-2012. Colector Omar Villerías.

Distribución: México.

Tribu Zygopini

Cylindrocopturus scaphiformis Champion, 1906. México: Michoacán: Morelia, Cerro del Águila, 20-VII-2013. $19^{\circ} 38^{\prime} 44^{\prime}$ 'N , $101^{\circ} 20^{\prime} 51^{\prime}$ 'O. 2288 msnm. Colector Macotulio Soto $\mathrm{H}$.

Distribución: Hondura, México.

Cylindrocopturs biradiatus Champion, 1906. México: San Luis Potosí: La Lugarda, Villa de Arriaga, 11-042014, $22^{\circ}$ 55' 34' 'N, $-101^{\circ} 55^{\prime} 34^{\prime}$ 'O. Colector Salvador Ordaz S.

Distribución: México.

Subfamilia Cossoninae

Tribu Onychopilini

Eurycorynes inaequalis (Champion, 1909). México: Veracruz: Xalapa, Bosque de Niebla, 3-IV-2014. 1,310 msnm. Colectado en madera en descomposición, colector Ma. Luisa Castillo y V. Mar Vélez.

Distribución: El Salvador, Guatemala, Honduras y México.

Subfamilia Cryptorhynchinae

Tribu Cryptorhynchini

Zascelis affaber (Boheman, 1844). México: Veracruz: Emiliano Zapata, carretera a Pinoltepec, 25-VII-2011. $19^{\circ}$ 27' 29' 'N, 096 46' 03' 'O. Colector Macotulio Soto H.

Distribución: México, Belice, Guatemala, Honduras, E1 Salvador, Nicaragua, Costa Rica, Panamá.

Zascelis brevicollis Champion, 1905. México: Veracruz: Hidalgotitlán. $5.5 \mathrm{~km}$. al NE de Hidalgotitlán, 27-X2012, $17^{\circ} 47^{\prime} 36^{\prime \prime} \mathrm{N}, 094^{\circ} 35^{\prime}$ 40' 'O. Colector Macotulio Soto $\mathrm{H}$.

Distribución: Nicaragua, Panamá.

Subfamilia Entiminae

Tribu Eustylini

Exophthalmus agrestis (Boheman, 1834). México: Veracruz: Hidalgotitlán, $5.5 \mathrm{~km}$ al NE de Hidalgotitlán, 27-X-2012. $17^{\circ} 47^{\prime} 36^{\prime \prime} \mathrm{N}, 094^{\circ} 35^{\prime}$ 40' 'O. Colector Macotulio Soto $\mathrm{H}$.

Distribución: México y Sudamérica.

Exophthalmus opulentus (Boheman, 1840). México: Veracruz: Hidalgotitlán, $5.5 \mathrm{~km}$ al NE de Hidalgotitlán, 27-X-2012. $17^{\circ} 47^{\prime} 36^{\prime}$ 'N, $094^{\circ} 35^{\prime}$ '40' 'O. Colector Macotulio Soto $\mathrm{H}$. 
Distribución: Guatemala, México.

Tribu Geonemini

Epicaerus aurifer Boheman, 1842. México: Guerrero: Cuetzala del Progreso, Chilacachapa, 14-VI-2012. Colector Omar Villerías. México: Guerrero: Acahuizotla, Las Bombas. 30-IX-2011. Colector Omar Villerías y E. A. López.

Distribución: México.

Epicaerus mexicanus Boheman, 1834. México: Guerrero: Cuetzala del Progreso, Chilacachapa, 20-VI-2012. Colector Omar Villerías.

Distribución: Estados Unidos de América, México. Claeoteges granulosa Champion, 1911. México: Veracruz: Totutla, El Mirador, 07-VII-2012. 19 $19^{\circ}$ ' $45^{\prime}$ 'N, 096 52' 57' O. 1057 msnm. Colector Macotulio Soto $\mathrm{H}$.

Distribución: Belice, Guatemala, Honduras, México. Tribu Naupactini

Ericydeus modestus (Gyllenhal, 1833). México: Guerrero: Cocula, La Perota, 01-XII-2012. Colector Omar Villerías.

Distribución: Estados Unidos de América, México.

Megalostylus dilaticollis Champion, 1911. México: Jalisco: La Huerta. Estación de Biología Chamela, 18-VII2013. 19 29' 36' 'N, 105 $03^{\circ}$ ' 21' 'O. Colector Macotulio Soto $\mathrm{H}$.

Distribución: México.

Megalostylus fusiformis Champion, 1911. México: Guerrero: Cuetzala del Progreso, Chilacachapa, 14-VII-2012. Colector Omar Villerías.

Distribución: México.

Pantomorus godmani (Crotch, 1867). México: Veracruz: Xalapa, Instituto de Ecología A. C. (INECOL), 13V-2013. Colector Macotulio Soto H.

Distribución: Nuevo Mundo, Las Antillas, Viejo Mundo.

Pantomorus picturatus Sharp, 1891. México: Jalisco:* La Huerta, Estación de Biología Chamela, Camino antiguo sur, 01-VIII-2012. 19 $29^{\circ} 36^{\prime}{ }^{\prime} \mathrm{N}, 105^{\circ} 02^{\prime} 21^{\prime}$ 'O. Colector Macotulio Soto H.

Distribución: Costa Rica, El Salvador, Guatemala, Honduras, México (Chiapas, Oaxaca, Jalisco).

Pantomorus viridicans Sharp, 1891. México: Guerrero: Cuetzala del Progreso, Chilacachapa, 20-VI-2012. Selva baja caducifolia. Colector Omar Villerías.

Distribución: México.

Phacepholis viridis Champion, 1911. México: Jalisco: Estación de Biología Chamela, 01-VIII-2012. 19² 29' $36^{\prime}$ 'N, $105^{\circ} 02^{\prime} 21^{\prime}$ 'O. Colector Macotulio Soto H.
Distribución: Estados Unidos de América y México.

Tribu Ophryastini

Ophryastes speciosus LeConte, 1853. México: Coahuila: Cuatrociénegas, 01-XI-2007. Colector García M. O con Red Entomológica.

Distribución: Estados Unidos de América, México.

Tribu Phillobiini

Aphrastus submarginatus Champion, 1911. México: Querétaro. Arroyo Seco, $1.5 \mathrm{~km}$ al NE de Ayutla, 26-VI2010. 1,130 msnm. Colector Macotulio Soto H.

Distribución: México.

Tribu Tanymecini

Pandeleteius macullicolis Champion, 1911. México: Michoacán. Morelia, Cerro del águila, 20-VII-2013. $19^{\circ} 38^{\prime}$ $44^{\prime}$ 'N, $101^{\circ} 20^{\prime} 51^{\prime}$ 'O. 2288 msnm. Colector Macotulio Soto $\mathrm{H}$.

Distribución: México.

Minyomerus laticeps (Casey, 1888). México: Coahuila: Cuatrociénegas, 22-VIII-2007. Colector García M. O.

Distribución: Estados Unidos de América y México.

Tanymecus confusus Say, 1831. México: Veracruz: Hidalgotitlán, $5.5 \mathrm{~km}$ al NE de Hidalgotitlán, 27-X-2012. $17^{\circ} 47^{\prime} 36^{\prime}$ 'N, $094^{\circ} 35^{\prime} 40^{\prime}$ 'O. Colector Macotulio Soto H. México: Veracruz: Xalapa, Las Ánimas. 07-VIII-2011. Colector Castillo M. L.

Distribución: Belice, Estados Unidos de América, Guatemala, México, Nicaragua.

Subfamilia Hyperinae

Tribu Ceperini

Phelypera distigma Jekel, 1865. México: Veracruz: Hidalgotitlán, $5.5 \mathrm{~km}$. Al NE de Hidalgotitlán, 27-X-2012. $17^{\circ} 47^{\prime} 36^{\prime}$ 'N, $094^{\circ} 35^{\prime}$ 40' 'O. Colector Macotulio Soto H. Distribución: Belice, Guatemala, México, Panamá.

Isorhinus depressus (Champion, 1902). México: Jalisco: La Huerta, Estación de Biología Chamela. 01-VIII-

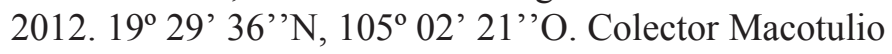
Soto H.

Distribución: Costa Rica, Guatemala, México.

Subfamilia Molytinae

Tribu Conotrachelini

Conotrachelus cristatus Fahraeus, 1837. México: Veracruz: Hidalgotitlán. $5.5 \mathrm{~km}$ al NE de Hidalgotitlán, 27-X2012. $17^{\circ} 47^{\prime} 36^{\prime \prime} \mathrm{N}, 094^{\circ} 35^{\prime}$ 40' O. Colector Macotulio Soto $\mathrm{H}$.

Distribución: Belice, Costa Rica, Guatemala, Honduras, México, Nicaragua, Panamá, Guadalupe y Sudamérica.

Tribu Cholinini

Neoerethistes cyanipes (Champion, 1903). México: Jalisco: La Huerta, Estación de Biología Chamela, 18-VII- 
2013. $19^{\circ} 29^{\prime} 47^{\prime}$ 'N, $105^{\circ} 02^{\prime}$ 24' 'O. 62 msnm. Colector Macotulio Soto $\mathrm{H}$.

Distribución: Costa Rica, México, Nicaragua, Sudamérica.

Tribu Hylobiini

Heilipodus dorbignyi (Guerin, 1844). México: Veracruz: Xalapa, Fraccionamiento Jacarandas. 02-IX-2011. Colector Macotulio Soto $\mathrm{H}$.

Distribución: Guatemala, México, Nicaragua, Panamá y Sudamérica.

Heilus caecus (Champion, 1902). Veracruz, Xalapa, Instituto de Ecología A.C., 11-XI-2013. Colector Macotulio Soto $\mathrm{H}$.

Distribución: México, Nicaragua.

Tribu Pissodini

Laccoproctus acalloides Boheman, 1843. México: Veracruz: Totutla, El Mirador, 07-VII-2012. 19 $12^{\circ}$ ' $52^{\prime}$ 'N, 096 53' 31' O. 1071 msnm. Colector Macotulio Soto H.

Distribución: Guatemala y México.

Tribu Sternechini

Sternechus extortus Chevrolat, 1833. México: Puebla: Cuetzalan, 20-VI-2012. Colector Omar Villerías.

Distribución: México (Durango, Oaxaca, Morelos, Veracruz, Guerrero).

Sternechus brevicolis Champion, 1902. México: Jalisco: La Huerta, Estación de Biología Chamela, Camino antiguo sur, 01-VIII-2012. 19 29'36'’ N, 105 02' 21'’O. Colector Macotulio Soto H.

Distribución: Guatemala, México, Nicaragua, Panamá.

\section{DISCUSIÓN}

México es uno de los países más ricos del mundo en especies de muchos grupos de plantas y animales. Aunque la riqueza específica de los curculiónidos mexicanos aun es desconocida. Según Ordoñez-Reséndiz (2005), Curculionidae es de las familias más estudiadas en México (incluyendo Platypodinae y Scolytinae). Sin embargo, los estudios de Curculionidae no se compara con los de Scarabaeidae o Melolontidae que, son los grupos más estudiados en el país (Michán \& Morrone 2002). Debido a la complejidad, diversidad, al reducido número de especialistas y falta de claves taxonómicas, aún existen limitantes para el estudio de Curculionoidea, ha habido avances en el conocimiento de los curculiónidos mexicanos (Anderson \& O'Brien 1996); aunque existe un gran rezago en la mayoría de los grupos, para los cuales, la principal referencia es la Biología Centrali-Americana (Sharp \& Champion
1889-1911), esto connota la imposibilidad de determinar a especie una fracción muy importante de curculiónidos, destacando ejemplares de los géneros Conotrachelus y Apion, para determinar las especies es necesario compararlas con ejemplares depositadas e identificadas en una colección de referencia, las colecciones más completas y curadas están en el extranjero.

Dryophthoridae y Curculionidae son de las grupos mejor conocido, el primero no es tan diverso como el último, ambos grupos con gran diversidad de especies en la región tropical y subtropical del mundo (Anderson 1993, 2002a). Por sus hábitos alimenticios, muchas especies tienen importancia económica, varias de estas son consideradas plagas agrícolas de una amplia variedad de plantas cultivadas y de productos almacenados (Muñiz 2001); en Dryophthoridae: Cactophagus validirostris (nopal, plátano), Metamasius hemipterus (caña de azúcar, plátano), Rhodobaenus sanguineus (girasol), Rhinostomus barbirostris (cocotero), Rhynchophorus palmarum (cocotero, papayo, plátano) Sphenophorus coesifrons (pastos), Scyphophorus acupunctatus se alimenta de diversas especies de agaváceas, actualmente genera importantes pérdidas económicas a la industria tequilera y mezcalera de México (Mendoza \& Martínez-Garza 2001, Solís et al. 2001, Romo \& Morrone 2012); Metamasius callizona, M. hemipterus y M. sellatus causan severos daños en bromelias. En Florida (USA), M. callizona y M. hemipterus representan las principales plagas de diversas especies de Tillandsia (Frank et al. 2006).

En Curculionidae: Optatus palmaris (chirimoya), Cylindrocopturus biradiatus (nopal), Epicaerus aurifer (alfalfa), E. mexicanus [cafeto, caña de azúcar, cítricos Woodruff (1985)], Exophthalmus opulentus (cítricos, caña de azúcar) y Pantomorus godmani, causa daños en diversas plantas ornamentales y cultivos agrícolas (Gyeltshen \& Hodges 2006). Para la mayoría de las especies de estos grupos se desconoce su biología, distribución geográfica y planta hospedera. En los últimos años se han hecho importantes contribuciones por conocer más a los curculiónidos: Maldonado et al. (2014) determinó el ciclo de vida de Optatus palmaris en Anonna muricata L, o Jones \& Zuñiga (2013) quienes han realizado un esfuerzo trascendental en la elaboración de una base de datos, fotografías y cartografía de los curculiónidos colectados y depositados en la Colección Entomológica de la Universidad Autónoma de Querétaro (UAQE). Esto puede facilitar el estudio taxonómico y biogeográfico de algunos grupos de picudos mexicanos.

Los curculíonidos identificados presentan una distribu- 
ción más hacia la región Neotropical (78\%), de éstos $26 \%$ con una distribución restringida a México, $74 \%$ a México y Centroamérica y, menos del 5\% con amplia distribución. Estos registros son similares a los reportados por SalasAraiza et al. (2001), Jones et al. (2005), Jones \& LunaCozar (2007). Aunque el conocimiento de la distribución geográfica de los picudos en el territorio mexicano aun es limitado, algunas especies han sido registradas más allá de la región biogeográfica de donde fue descrita: Myrmex cylindricollis y Onychylis texanus, M. cylindrocollis especie registrada para Córdoba y Actopan. Veracruz, México (Champion 1902-1906), ha sido colectado (norte del país) en la Reserva de la Biosfera El Cielo (RBC), Tamaulipas. Por otro lado, O. texanus (colectado en el sur del país) solo se conocía en Texas, U.S.A. (Burke 1959), Burke (1961) menciona que observó un espécimen de Tehuantepec, Oaxaca con caracteres similares a $O$. texanus. No confirmó si era esta especie. Es imperativo denotar que el suroeste de los Estados Unidos de América, Tamaulipas y Veracruz forma parte Zona de Transición Mexicana (ZTM), ésta área ha sido definida como compleja y variada donde se superponen biotas Neárticas y Neotropicales, haciendo de la ZTM un área de gran importancia biogeográfica y biológica (Halffter 1976, Morrone 2005).

AGRADECIMIENTOS. Al Consejo Nacional de Ciencia y Tecnología (CONACYT) por el apoyo otorgado a través del programa Ciencia Básica 2011 (Proyecto No. 169604). Al Instituto de Ecología, A. C. (INECOL), al Departamento de Parasitología Agrícola de la Universidad Autónoma Agraria Antonio Narro (UAAAN), al Dr. Santiago Zaragoza y M.C. Cristina Mayorga de la Colección Nacional de Insectos (CNIN), al Dr. Robert W. Jones y M.C. Alejandro Zúñiga de la Colección Entomológica de la Universidad Autónoma de Querétaro (UAQE) por los apoyos y facilidades otorgadas al primer autor. A Adriana Rojas Hernández, Julio Cesar Chacón Hernández, Omar Villerías Simbron y Sandra María Guzmán Martínez por el apoyo en la recolecta de curculiónidos.

\section{LITERATURA CITADA}

Alonso-Zarazaga, M. A. \& Lyal, C. H. C. 1999. A world catalogue of families and genera of Curculionoidea (Insecta: Coleoptera) (Excepting Scolytidae and Platypodidae). The Natural History $\mathrm{Mu}-$ seum, London y Museo Nacional de Ciencias Naturales (CSIC), Entomopraxis, Barcelona, España. 315 pp.

Anderson, R. S.1993. Weevils and plants: Phylogenetic versus ecological mediation of evolution of host plant associations in Curculioninae (Coleoptera: Curculionidae). Memoirs of the Entomological Society of Canada, 165: 197-232.

Anderson, R. S. 2002. Family 131. Curculionidae Latreille 1802. Pp. 722-806. In: R. H. Arnett, Jr., M. C. Thomas, P. E. Skelley \& F. J.
Howard (Eds.). American Beetles, Volume II: Polyphaga: Scarabaeoidea through Curculionoidea. CRC Press LLC, Boca Raton.

Anderson, R. S. 2002a. The Dryophthoridae of Costa Rica and Panama: Checklist with keys, new synonymy and descriptions of new species of Cactophagus, Mesocordylus, Metamasius and Rhodobaensus (Coleoptera; Curculioniodea). Zootaxa, 80: 1-94.

Anderson, R. S. \& O'Brien, C. W. 1996. Curculionidae (Coleoptera). Pp. 329-350. In: J. Llorente-Bousquets, A. N. García-Aldrete \& E. González-Soriano (Eds.). Biodiversidad, Taxonomía y Biogeografía de Artrópodos de México: Hacia una sintesis de su conocimiento. Universidad Nacional Autónoma de México, Instituto de Biología, México, D.F.

Barrigan-Tuñon, J. E. 2009. Coleoptera Tropical, Curculionidae. Available at: http://coleoptera-neotropical.org/paginas/6-famneo/ Curculionoidea/Curculionidae_fam.html (accessed on July 2014).

Burke, H. R. 1959. Notes on Some Texas Curculionidae with a Description of a New Species. The Coleopterists Bulletin, 13: 36-41.

Burke, H. R. 1961. Notes on Onychylis LeConte with Descriptions of two new species (Curculionidae). The Coleopterists Bulletin, 15: 1-8.

Champion, G. C. 1902-1906. Insecta. Coleoptera. Rhynchophora, Curculionidae, Curculioninae (part). Pp. 1-713. In: R. H. Porter (Eds.). Biologia Centrali-Americana. Vol. 4. Part 4. London.

Champion, G. C. 1909-1911. Insecta, Coleoptera, Rhynchophora, Curculionidae, Curculioninae, (concluded) and Calandrinae. Pp 1-213. In: R. H. Porter (Eds.). Biologia Centrali-Americana. Vol. IV. Pt.7. London

Clark, W. E. 1978. The weevil genus Sibinia Germar: Natural History, Taxonomy, Phylogeny, and Zoogeography, with revision of the New World species (Coleoptera: Curculionidae). Quaestiones Entomologicae, 14: 91-387.

Clark, W. E. 1987. The species of Anthonomus in the Albolineatus group (Coleoptera: Curculionidae) Transactions of the American Entomological Society, 113: 309-359.

Clark, W. E. 1989. Revision of the Neotropical weevil genus Atractomerus Duponchel and Chevrolat (Coleoptera: Curculionidae). American Entomological Society, 115: 313-414.

Clark, W. E. 1990. Revision of the fischeri Species Group of the Genus Anthonomus Germar (Coleoptera: Curculionidae). Transactions of the American Entomological Society, 116: 619-642.

Clark, W. E. 1993. The Anthonomus ocularis species group (Coleoptera: Curculionidae). Transactions of the American Entomological Society, 119: 95-130.

Clark, W. E. \& Burke, H. R. 2005. Revision of the genus Cnemocyllus Dietz of the weevil genus Anthonomus Germar (Coleoptera: Curculionidae, Anthonomini). Insecta Mundi, 19: 1-55.

Frank, J. H., Cooper, T. M. \& Larson, B. C. 2006. Metamasius callizona (Coleoptera: Dryophthoridae) longevity and fecundity in the laboratory. Florida Entomologist, 89: 208-212.

Franz, N. M., Chamorro, M. L, Fleming, D., Setliff, G. P., Fleming, D. \& O'Brien, C. W. 2012. Collaborative Research Digitization TCN: Southwest Collections of Arthropods Network (SCAN). Available at: http://symbiota4.acis.ufl.edu/scan/portal/checklists/ checklist.php?cl=1 (accessed on July 2014).

Fred. T. 2009. Entomofauna Guyane. Available at: http://entomofauna-guyane.fr/?q=category/114-curculionoidea-latreille-1802 (accessed on July 2014). 
Gyeltshen, J. \& Hodges, A. 2006. Fuller Rose Beetle, Naupactus godmanni (Crotch) (Insecta: Coleoptera: Curculionidae). Institute of Food and Agricultural Sciences (University of Florida. Gainesville, FL, 32611: 1-5.

Halffter, G. 1976. Distribución de los insectos en la Zona de Transición Mexicana: relaciones con la Entomofauna de Norteamérica. Folia Entomológica Mexicana, 35:1-64.

Jones, R. W. \& Zuñiga, O. A. J. 2013. Captura de datos de la Colección de Curculionoidea (Insecta: Coleoptera) de la Universidad Autónoma de Querétaro. Universidad Autónoma de Querétaro. Facultad de Ciencias Naturales. Informe final SNIB-CONABIO proyecto No. HC010. México, D. F. 12 pp.

Jones, R. W. \& Luna-Cozar, J. 2007. Lista de las especies de Curculionoidea (Insecta: Coleoptera) del estado de Querétaro, México. Acta Zoológica Mexicana (n.s.), 23: 59-77.

Jones, R. W., Maldonado-Niño, S. \& O'Brien, C. W. 2005. 39. Coleoptera: Curculionidae. Pp. 426-437.

Sánchez-Ramos, G., Reyes-Castillo, P. \& Dirzo, R. (Eds.). In: Historia Natural de la Reserva de la Biosfera El Cielo, Tamaulipas. México. México. Universidad Autónoma de Tamaulipas. Impreso en Hong Kong.

Kissinger, D. G. 1964. Curculionidae of America North of Mexico. A key to the Genera. Taxonomic Publication, South Lancaster Massachusetts. $133 \mathrm{pp}$.

Kissinger, D. G. 1970. Curculionidae Tribe Ophryastini of North America (Coleoptera). Taxonomic Publication, South Lancaster, Massachusetts. 238 pp.

Llorente-Bousquets, J., García-Aldrete, A. N. \& González-Soriano, E. 1996. Biodiversidad, Taxonomía y Biogeografía de Artrópodos de México: hacia una síntesis de su conocimiento. Universidad Nacional Autónoma de México, México, D. F. 660 pp.

Maldonado J. E., Hernández F. L. M., Luna E. G., Gómez A. J.R., Flores C. R. J., \& Orozco S. M. 2014. Bioecology of Optatus palmaris Pascoe (Coleoptera: Curculionidae) in Annona muricata L. Southwestern Entomologist, 39: 773-782. doi: http://dx.doi. org/10.3958/059.039.0408

Michán, L. \& Morrone, J. J. 2002. Historia de la Taxonomía de Coleoptera en México durante el Siglo XX: Una primera aproximación. Folia Entomológica Mexicana, 41: 67-103.

Morrone, J. J. 2005. Hacia una síntesis biogeográfica de México. Revista Mexicana de Biodiversidad, 76: 207-252.

Morrone, J. J. 2014. Biodiversidad de Curculionoidea (Coleoptera) en México. Revista Mexicana de Biodiversidad, suplemento 85: 312-325.

Muñiz-Vélez, R. 2001. Algunos Curculiónidos en las plantas cultivadas en México. Boletín de la Sociedad Mexicana de Entomología, 16:1-14.

O'Brien, C. W. \& Wibmer, G. J. 1982. Annotated checklist of the weevils (Curculionidae sensu lato) of North America, Central America and the West Indies (Coleoptera: Curculionidae). $\mathrm{Me}$ moirs of the American Entomological Institute, 34: 382 pp.

O'Brien, C. W. \& Wibmer, G. J. 1984. Annotated checklist of the weevils (Curculionidae sensu lato) of North America, Central America and the West Indies-Supplement 1. Southwestern Entomological, 9: 286-307.

O'Brien, C. W. \& Wibmer, G. J. 1990. Taxonomic Revision of the New World Genera of the tribe Stenopelmini. Entomological-Biological Control, Florida A\&M University. Tallahassee. 76 pp.
Ordoñez-Reséndiz, M. M. 2005. Colección de Coleoptera (Insecta) de la Facultad de Estudios Superiores Zaragoza, UNAM. Acta Zoológica Mexicana (n.s.), 21: 95-106.

Perkins. P. 2010. Museum of Comparative Zoology. The Entomology Type Collection. Available at: http://140.247.119.225/mcz/recordlist.php (accessed on July 2014).

Prena, J. 2005. The Middle American species of Embates Chevrolat (Coleoptera: Curculionidae: Baridinae). Zootaxa, 1100: 1-151.

Prena, J. 2009. A review of the species of Geraeus Pascoe and Linogeraeus Casey found in the Continental United States (Coleoptera: Curculionidae: Baridinae). The Coleopterists Bulletin, 63(2): 123-172.

Romo, A. \& Morrone, J. J. 2012. Especies mexicanas de Curculionidae (Insecta: Coleoptera) asociadas con agaves (Asparagaceae: Agavoideae). Revista Mexicana de Biodiversidad, 83: 10251035.

Rosas, E. M. V. 2011. Análisis filogenético del género Pantomorus Schoenherr (Coleoptera: Curculionidae: Entiminae). Tesis de Doctorado. Facultad de Ciencias. Universidad Nacional Autónoma de México. D. F. 74 pp.

Salas-Araiza, M. D., O'Brien C. W. \& Romero-Nápoles, J. 2001. Curculionoidea (Insecta: Coleoptera) from the State of Guanajuato, Mexico. Insecta-Mundi, 15(1): 45-57.

Sharp, D. \& Champion, G. C. 1889-1911. Coleoptera, Rhynchophora. Curculionidae, Attelabinae. Pterocolinae, Allocoryninae, Apioninae, Thecesterninae, Otiorhynchinae. Pp 1-345. In: R. H. Porter (Eds). Biologia Centrali-Americana. Vol. 4, part 3. London.

Solís-Aguilar, J. F., González-Hernández, H., Leyva-Vásquez, J. L., Equihua-Martínez, A., Flores-Mendoza, F. J. \& Martínez-Garza, A. 2001. Scyphophorus acupunctatus Gyllenhal, plaga del agave tequilero en Jalisco México. Agrociencia, 35: 663670 .

Soto-Hernández, M., Jones R. W. \& Reyes-Castillo, P. 2013. A key to the Mexican and Central America Genera of Anthonomini (Curculionidae, Curculioninae). ZooKeys, 260: 31-47.

Vaurie, P. 1951. Revision of the genus Calendra (formerly Sphenophorus) in the United States and Mexico (Coleoptera, Curculionidae). Bulletin of the American Museum of Natural History, 98: 29-186.

Vaurie, P. 1966. A Revision of the Neotropical Genus Metamasius (Coleoptera, Curculionidae, Rhynchophorinae), Species Groups I and II. Bulletin of the American Museum of Natural History, 131: 211-338.

Vaurie, P. 1967. A revision of the Neotropical Genus Metamasius (Coleoptera, Curculionidae, Rhynchophorinae). Bulletin of the American Museum of Natural History, 136: 175-268.

Vaurie, P. 1978. Revision of the Genus Sphenophorus in South America (Coleoptera, Curculionidae, Rhynchophorinae). American Museum Novitates (American Museum of Natural History), 2656: $1-30$.

Vaurie, P. 1981. Revision of Rhodobaenus. Pt. 2. Species in North America (Canada to Panama) (Coleoptera, Curculionidae, Rhyncophorinae). Bulletin of the American Museum of Natural History, 171: 121-197.

Woodruff, R. E. 1985. Citrus Weevils in Florida and the West Indies: Preliminary Report on Systematics, Biology, and Distribution (Coleoptera: Curculionidae). The Florida Entomologist, 68: 370-379. 